\title{
The Severe Acute Respiratory Syndrome Coronavirus-2 Pandemic: An Overview to Control Human-wildlife and Human-human Interactions
}

\author{
Ahmed E. Taha ${ }^{1,2}$ (iD) \\ ${ }^{1}$ Microbiology and Immunology Unit, Department of Pathology, College of Medicine, Jouf University, \\ Al-Jouf, Saudi Arabia. ${ }^{2}$ Medical Microbiology and Immunology Department, Faculty of Medicine, \\ Mansoura University, Mansoura, Egypt.
}

\begin{abstract}
Prevention of high-risk human-wildlife and human-human interactions, which can accelerate the occurrence of future outbreaks, epidemics or pandemics, requires global efforts. These efforts should focus on reducing the risk of contact with wildlife, pre-outbreak surveillance of wildlife and high-risk people, improvement of wildlife trade biosafety, and learning lessons from the previous attacks. International collaborations, building on the experiences learnt during facing the attacks by severe acute respiratory syndrome-coronavirus (SAR-CoV), Middle East respiratory syndrome-coronavirus (MERS-CoV) and severe acute respiratory syndrome coronavirus-2 (SARS-CoV-2), will accelerate implementation of novel anti-viral prevention and control measures to avoid occurrence of such devastating viral outbreaks, epidemics and pandemics in the future.
\end{abstract}

Keywords: COVID-19, MERS-CoV, nCoV, SARS-CoV, Surveillance

*Correspondence: drahmadmicro@yahoo.com; aeattia@ju.edu.sa

(Received: April 09, 2020; accepted: May 18, 2020)

Abbreviations: COVID-19: coronavirus disease-19; MERS-CoV: Middle East respiratory syndrome-coronavirus; nCoV: novel coronavirus; SARS-CoV: severe acute respiratory syndrome-coronavirus; SARS-CoV-2: severe acute respiratory syndrome coronavirus-2.

Citation: Taha AE. The Severe Acute Respiratory Syndrome Coronavirus-2 Pandemic: An Overview to Control Human-wildlife and Human-human Interactions. J Pure Appl Microbiol. 2020;14(2):1095-1098. doi: 10.22207/JPAM.14.2.02

(C) The Author(s) 2020. Open Access. This article is distributed under the terms of the Creative Commons Attribution 4.0 International License which permits unrestricted use, sharing, distribution, and reproduction in any medium, provided you give appropriate credit to the original author(s) and the source, provide a link to the Creative Commons license, and indicate if changes were made. 


\section{INTRODUCTION}

The current pandemic of the novel coronavirus, '2019-nCoV,' that started in China (Wuhan, December 27, 2019), has highlighted our continued global susceptibility to epidemics and pandemics. The International Committee on Taxonomy of Viruses named it 'the severe acute respiratory syndrome coronavirus-2 (SARSCoV-2)' and the disease referred to as 'coronavirus disease-19 (COVID-19).' The majority of the early cases started in a seafood market and were related to wildlife. This virus seems to originate from bats and appeared in the wildlife market by animals which represented the intermediate hosts of this virus and further spread by interaction with humans ${ }^{1}$.

Chinese scientists detected this new syndrome, isolated its causative virus, and reported the viral genetic sequence to the world within two weeks ${ }^{2}$. However, even with these efforts, this novel virus has rapidly spread through infected persons traveling within China and internationally. The economy of China has expanded after the end of the severe acute respiratory syndrome - coronavirus (SARS-CoV) epidemic in 2003, and many people became wealthier, resulting in increased domestic and international travel. Furthermore, as part of China's expansion of trade and business, there has been an upsurge in travel between China and African countries, the New World, and Southeast Asia. This generally clarifies why the virus was able to spread out of China much more rapidly than SARS-CoV, which needed more than two months to reach Hong Kong, and then other areas of the world ${ }^{3}$. China, Southeast Asia, sub-Saharan Africa, South Asia, and Latin America are high-risk areas for outbreaks ${ }^{4}$.

At this time, four key steps are mandatory for reducing the risk of future outbreaks, epidemics and pandemics similar to those resulting from the SARS-CoV and SARS-CoV-2:

\section{Reduction in contact risk with wildlife}

Wildlife trade has played a key role in the worldwide appearance of SARS-CoV-2 and many other fatal pathogens (e.g., SARS-CoV in China, Ebola in Africa, and monkeypox in the USA, and salmonellosis in Europe and the USA). In addition, wildlife trade is a major risk for exposing people to many pathogens that have not yet been discovered, leading to the next potential outbreak. This makes wildlife trade an important target for prevention and control policies to avoid forthcoming epidemics and pandemics ${ }^{5}$.

Reducing the contact risk with wildlife is not an easy task and it is challenging to modify behaviors, which are influenced by local traditions and culture. Furthermore, the consumption of rare expensive wildlife products is considered an indicator of wealth. Modifying these traditional and cultural habits requires time; however, data from behavioral questionnaires suggest a reduction in wildlife consumption in younger generations ${ }^{6}$.

Pre-outbreak surveillance strategy of wildlife and at-risk people for high-risk viruses

In China, there are many coronaviruses in the wildlife that represent a major potential source of human infection. In addition, thousands of other coronaviruses in bats all over Southeast Asia are estimated to have epidemic and pandemic potential. It is necessary for scientists in these high-risk countries to detect these viruses, catalog them, develop a reference library for rapid viral diagnosis, and try to use effective vaccines and therapeutic drugs against them?

People frequently contacting wild or domestic animals during their work, and patients suffering from influenza-like illness or acute respiratory infection with unknown cause should be included in a surveillance program designed by local public health authorities, as a costeffective system to detect human infection by novel viruses ${ }^{8}$. This surveillance program can be synchronized with many sectors of healthcare, public health, forestry, agriculture, and research institutions to implement sample collection and testing of wildlife, domestic animals, and people. Evidence-based policies to decrease risks should then be planned and implemented in societies where viral spillover is detected ${ }^{9}$.

\section{Improvement of wildlife trade biosafety}

The biosafety of markets needs improvement in the context of the source animals traded, sanitation and hygiene facilities, and regulations. During the outbreak, wildlife trade must be temporarily banned in some provinces, and/or nationally. Farmed animals represent 
a lower risk than wild animals in the regard. However, routine veterinary care and disease surveillance at the farms and during transport to markets must be improved ${ }^{5}$.

Learning lessons from previous outbreaks, epidemics and pandemics

The lessons learnt from facing SARS-CoV,

Middle East respiratory syndrome- coronavirus (MERS-CoV), and SARS-CoV-2 attacks can provide valuable insight into an ideal way to handle similar attacks in the future. These include preventing direct contact with suspected animal reservoirs, proper hand hygiene, isolation of suspected individuals, and isolation of infected patients in a negative pressure room. The rapid sharing of viral genetic sequences will lead to faster diagnosis on a global scale. Transparency must be present between governments and the public, which in turn will limit the rapid viral spread. Precautionary measures to reduce the risk of importing of a novel infectious virus must be adhered to by airport authorities around the globe. Screening of air passengers coming from high-risk areas and reporting of patients with respiratory symptoms to the authorities are obligatory for containing the rapid spread of cases. After further investigation, travelers that fit a novel virus case definition must be sent to local hospitals for further management. However, this may be difficult because of the asymptomatic nature of many cases. Moreover, airport authorities must display alerts about the clinical picture of the novel virus and its preventive measures to be followed globally by travelers ${ }^{10}$.

The lessons learnt during SAR-CoV and MERS-CoV vaccine development trials indicate that several approaches can be tried during SARSCoV-2 vaccine development, but we should wait not less than six months after starting clinical trials in humans. Meaning that, this prospective vaccine could come too late to affect the early waves of the attack and might be beneficial only against the post-pandemic waves or if SARS-CoV-2 remains as a seasonal virus. Nowadays might be the best period to think through investing in vaccines against emerging viruses using out-of-thebox novel approaches involving well-developed emergency plans that can be activated in the event of new emerging viruses to allow development, testing, production and distribution of vaccines swiftly within few weeks, not months or years. One approach is to develop broadly protective vaccine covering all coronaviruses or at least beta coronaviruses ${ }^{11}$. This approach is enduring against influenza viruses ${ }^{12}$. An alternative but challenging approach would be the surveillance in the animal reservoirs to isolate the highly suspected viruses that are likely to cause human respiratory diseases with the potential to cause pandemics. Vaccines depending on using these viral isolates, could be produced and tested in animals to determine their protection mechanisms. At this moment, these vaccines could be ready to move to the stages of clinical trials in humans as soon as needed. These expensive out-of-the-box approaches require global vision and political will as well as harmonization between governments, pharmaceutical companies and the World Health Organization (WHO) to avoid the harmful effects of such emerging viruses on the global populations as well as the global economy if reaching the pandemic stage ${ }^{11}$.

\section{CONCLUSION}

Expanding human activities on a global scale has led to an exponential upsurge in the rate of human-animal contact and the possibility of novel pandemics are expected to be more frequent and devastating in the future. Facing the risk of future epidemics and pandemics requires global efforts. More research and collaboration among scientists from all over the world should be directed towards the development of effective methods for pre-outbreak surveillance to predict the disease with early and timely diagnosis and treatment of suspected cases to reduce morbidity and mortality. Novel anti-viral prevention and control measures are urgently needed to avoid occurrence of such fatal outbreaks, epidemics or pandemics in the future by building on the experiences learnt during facing SAR-CoV, MERSCoV and SARS-CoV-2 attacks.

\section{ACKNOWLEDGEMENTS}

I would like to thank Editage (www. editage.com) for English language editing.

\section{FUNDING}

None. 


\section{ETHICS STATEMENT}

This article does not contain any studies with human participants or animals performed by the author.

\section{DATA AVAILABILITY}

All datasets generated or analyzed during this study are included in the manuscript.

\section{REFERENCES}

1. Zhou $P$, Yang $X-L$, Wang $X-G$, et al. A pneumonia outbreak associated with a new coronavirus of probable bat origin. Nature. 2020;579:270-273. https://doi.org/10.1038/s41586-020-2012-7

2. Zhu N, Zhang D, Wang W, et al. A novel coronavirus from patients with pneumonia in China, 2019. N Engl J Med. 2020;382:727-733. https://doi.org/10.1056/ NEJMoa2001017

3. The 2019-nCoV Outbreak Joint Field Epidemiology Investigation Team, Li Q, An outbreak of NCIP (2019nCoV) infection in China- Wuhan, Hubei Province, 2019 -2020. China CDC Weekly. 2020;2(5):79-80.

4. Allen T, Murray KA, Zambrana-Torrelio C, et al. Global hotspots and correlates of emerging zoonotic diseases. Nat Commun. 2017;8:1124. https://doi.org/10.1038/ s41467-017-00923-8

5. Li H-Y, Zhu G-J, Zhang Y-Z, et al. A qualitative study of zoonotic risk among rural communities in Southern
China. International Health. 2020;12:77-85. https:// doi.org/10.1093/inthealth/ihaa001

6. Zhang $L$, Yin F. Wildlife consumption and conservation awareness in China: a long way to go. Biodivers Conserv. 2014;23:2371-2381. https://doi.org/10.1007/s10531014-0708-4

7. Carroll D, Daszak P, Wolfe ND, et al. The global virome project. Science. 2018;359(6378):872-874. https://doi. org/10.1126/science.aap7463

8. Olival KJ, Hosseini PR, Zambrana-Torrelio C, Ross N, Bogich TL, Daszak P. Host and viral traits predict zoonotic spillover from mammals. Nature. 2017;546:646-650. https://doi.org/10.1038/nature22975

9. Gao GF. From "A" IV to "Z" IKV: attacks from emerging and re-emerging pathogens. Cell. 2018;172(6):11571159. https://doi.org/10.1016/j.cell.2018.02.025

10. Peeri NC, Shrestha N, Rahman MS, et al. The SARS, MERS and novel coronavirus (COVID-19) epidemics, the newest and biggest global health threats: what lessons have we learned? Int J Epidemiol. 2020; pii: dyaa033. https://doi.org/10.1093/ije/dyaa033

11. Amanat F, Krammer F. SARS-CoV-2 Vaccines: Status Report. Immunity Perspective, 2020;52(4): 583-589. https://doi.org/10.1016/j.immuni.2020.03.007.

12. Erbelding EJ, Post DJ, Stemmy EJ, et al. A Universal Influenza Vaccine: The Strategic Plan for the National Institute of Allergy and Infectious Diseases. J Infect Dis, 2018;218(3): 347-354. https://doi.org/10.1093/ infdis/jiy103. 